Frequency-dependent orthotropic damping properties of Nomex honeycomb composites

Yong Zhou, Anna Liu, Yongzheng Xu, Yunli Guo, Xiaosu Yi, Yuxi Jia

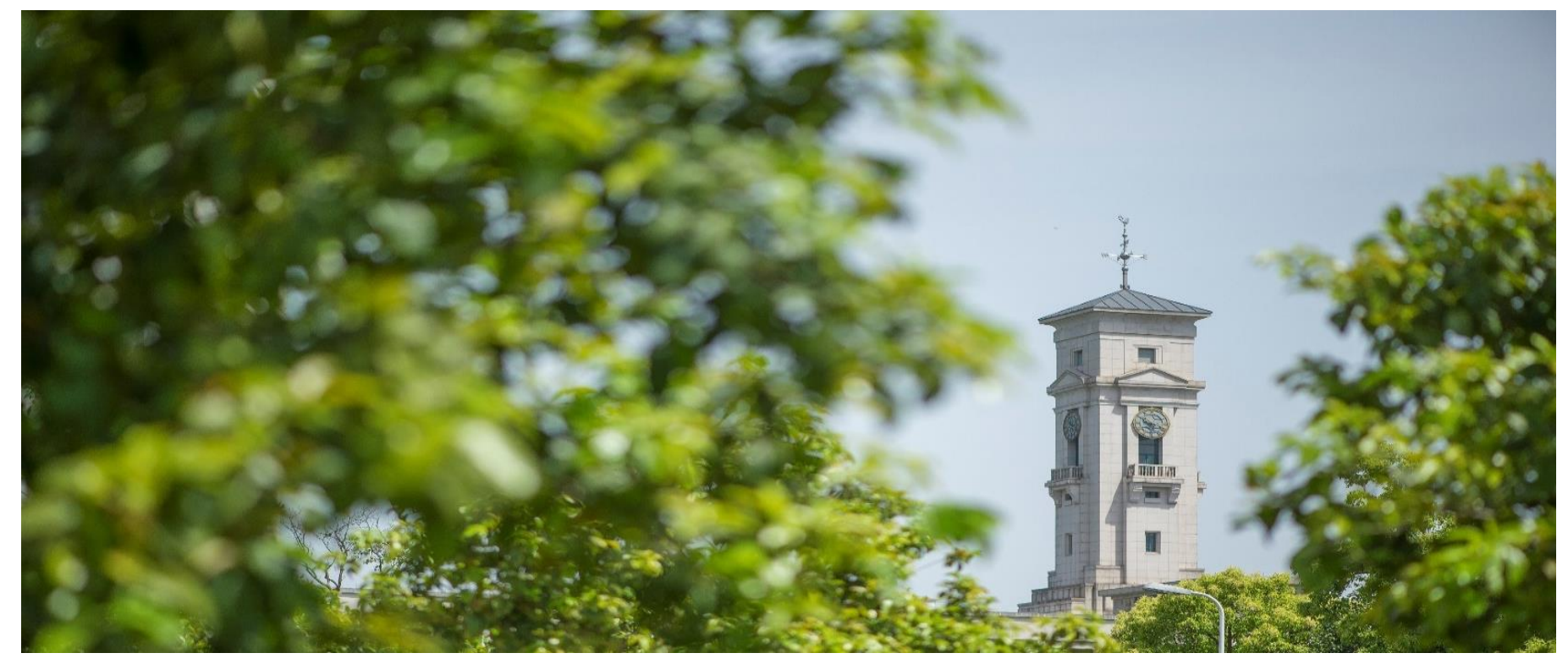


University of Nottingham Ningbo China, 199 Taikang East Road, Ningbo, 315100, Zhejiang, China.

First published 2020

This work is made available under the terms of the Creative Commons Attribution 4.0 International License:

http://creativecommons.org/licenses/by/4.0

The work is licenced to the University of Nottingham Ningbo China under the Global University Publication Licence:

https://www.nottingham.edu.cn/en/library/documents/research/global -university-publications-licence-2.0.pdf 


\title{
Frequency-dependent orthotropic damping properties of Nomex honeycomb
} composites

\author{
Yong Zhou ${ }^{\mathrm{a}}$, Anna Liu ${ }^{\mathrm{a}}$, Yongzheng Xu ${ }^{\mathrm{a}}$, Yunli Guo ${ }^{\mathrm{a}}$, Xiaosu Yib ${ }^{\mathrm{b}}$, Yuxi Jia ${ }^{\mathrm{a}, *}$ \\ ${ }^{\text {a }}$ Key Laboratory for Liquid-solid Structural Evolution \& Processing of Materials \\ (Ministry of Education), Shandong University, Jinan 250061, China \\ ${ }^{b}$ Faculty of Science and Engineering, University of Nottingham Ningbo China, Ningbo \\ 315100, China \\ *Corresponding author: jia_yuxi@sdu.edu.cn
}

\begin{abstract}
In this paper, the orthotropic damping behavior of Nomex honeycomb composites and its causes are investigated. The needed specimen sizes for the measurement of the frequency-dependent transverse shear moduli (TSM) and fundamental damping coefficients of the honeycomb cores were analyzed at first. Then, the effects of cell side length and beam orientation on the orthotropic damping properties were explored. The results reveal that relatively high TSM $\left(G_{\mathrm{LT}}\right)$ and damping values $\left(\eta_{\mathrm{WT}}\right)$ can be obtained by decreasing the cell side length without adding any additional weight. Damping mechanism analysis indicates that the difference in damping contribution of the interfacial phase to honeycomb core in different directions leads to the orthotropic damping behavior of honeycomb core. This study is helpful to guide the TSM measurement and structure design of honeycomb composites.
\end{abstract}

Keywords: Honeycomb composite; Experimental/numerical method; Orthotropic damping property; Transverse shear modulus; Cell side length 


\section{Introduction}

Nomex honeycomb core has been used as a crucial component in weight-sensitive fields such as aerospace and transport packaging, because of its relatively high damping properties, excellent out-of-plane specific stiffness, and low dielectric properties [1-3]. Among all its dominant properties, the relatively high damping properties are of prime importance in the noise and vibration control [4], which improve comfort. Due to the unique close-packed hexagonal structure, both of the mechanical and damping properties of the Nomex honeycomb core show orthotropic anisotropy. However, the latter and its causes are rarely reported. Considering that the Nomex honeycomb core consists of a series of polymer materials whose damping properties usually exhibit frequency dependence, the frequency-dependent orthotropic damping properties of honeycomb composites are explored in this paper.

The accurate acquisition of the frequency-dependent fundamental damping coefficients of the honeycomb core is an important prerequisite for exploring the orthotropic damping behavior of honeycomb composites. Since the honeycomb cores are usually relatively soft and cannot be tested by the vibration testing directly, limited works [5-8] have been done to obtain the damping values of these non-self-supporting honeycomb cores. Adams and Bacon [8] tested the damping values of aluminum and Nomex honeycomb cores directly with the improved homemade equipment. The results showed that the transverse shear damping coefficients in planes $(\mathrm{L}, \mathrm{T})$ and $(\mathrm{W}, \mathrm{T})$ are different. Nevertheless, the effect of frequency on these damping values was not discussed. Dynamic mechanical analysis [9] can obtain the damping values under continuous frequency directly, but the strict requirement of specimen size and limited 
testing range of frequency restrict the usage of this method on the honeycomb core. More studies focused on the indirect method. For example, several works $[5,6]$ obtained the damping values of honeycomb cores by means of the experimental/numerical method (fitting the calculated and experimental modal loss factors of sandwich structures).

Similar to the results obtained by Adams and Bacon, the damping values of honeycomb core exhibits orthotropic anisotropy. The advantage of this strategy is that the calculation efficiency is relatively high; however, some of the obtained damping values lack further sufficient experimental verification.

Based on the aforementioned experimental/numerical method, the damping values of T722 Nomex honeycomb cores with different phenolic resin thicknesses were obtained and analyzed in our previous study [10], where the damping parameters were validated. The orthotropic damping behavior of the honeycomb core has also been observed, but its cause has not been explored. Furthermore, the results showed that the damping value of the phenolic resin was relatively low; nevertheless, the damping contributions of Nomex paper and interfacial phase remain unclear.

In order to investigate the orthotropic damping behavior and its causes of honeycomb composites, and avoid most of the limitations faced in the above-mentioned works, the frequency-dependent damping values of the honeycomb cores are derived from the bending modal loss factors of aluminum/honeycomb sandwich beams in the ribbon (L) and cross (W) directions. Subsequently, these values are validated with the honeycomb sandwich beam and panel. Then, the effect of structural factor (cell side length) is studied to further reveal the damping mechanism. Inspired by the studies of obtaining the relative high damping properties of laminated composites through adjusting the fiber orientation 
[11], the potential application value of orthotropic damping properties of honeycomb cores in the obtaining of relative high structure-damping performance of honeycomb sandwich composites deserve further study. Therefore, the effect of beam orientation on the anisotropic damping properties of honeycomb sandwich structure is explored based on the verified TSM and damping coefficients of the honeycomb core at last.

\section{Experiment}

\subsection{Materials and fabrication}

Considering that the honeycomb cores with cell side lengths of $1.83 \mathrm{~mm}$ and $2.75 \mathrm{~mm}$ are relatively more widely used in engineering, NH-1-1.83-48 and NH-1-2.75-48 (AECC Beijing Institute of Aeronautical Materials) are investigated in this paper. They have the same Nomex paper of X612 (X-FIPER New Material Co., Ltd) and volume density of 48 $\mathrm{kg} / \mathrm{m}^{3}$. Aluminum (type 1060) skins are used for all sandwich beams. In addition, carbon fiber reinforced polymer (CFRP)/honeycomb sandwich panel in the sequence of [45/45/45/-45/honeycomb core/-45/45/-45/45] is prepared to validate the obtained properties of the honeycomb core. The basic properties of involved skins are listed in Table 1, where the properties of aluminum skin refer to the aluminum alloy manual [12], and the tensile moduli and shear moduli of the CFRP skin are tested based on GB/T 3354-2014 and GB/T 3355-2014, respectively.

Table 1 Properties of aluminum and unidirectional CFRP skins.

\begin{tabular}{cccccccc}
\hline \multirow{2}{*}{ Material } & $\begin{array}{c}E_{11} \\
\end{array}$ & $E_{22}, E_{33}$ & $G_{12}, G_{13}$ & $G_{23}$ & $v_{12}, v_{13}$ & $v_{23}$ & $\begin{array}{c}\rho \\
\end{array}$ \\
& $(\mathrm{GPa})$ & $(\mathrm{GPa})$ & $(\mathrm{GPa})$ & $(\mathrm{GPa})$ & & & $\left(\mathrm{kg} / \mathrm{m}^{3}\right)$ \\
\hline Aluminum & 69 & 69 & 25.94 & 25.94 & 0.33 & 0.33 & 2705 \\
CFRP & 133 & 5.91 & 4.72 & 4.5 & 0.30 & 0.35 & 1560 \\
\hline
\end{tabular}


Unidirectional $\left(\left[0^{\circ}\right]_{16}\right)$ and orthogonal $\left(\left[ \pm 45^{\circ}\right]_{4 \mathrm{~s}}\right)$ CFRP laminates were fabricated at $120^{\circ} \mathrm{C}$ for $1.5 \mathrm{~h}$ under $0.7 \mathrm{MPa}$ after the pre-curing process of $0.5 \mathrm{~h}$ at $85^{\circ} \mathrm{C}$. The $\mathrm{L}-$ or $\mathrm{W}$ direction aluminum/honeycomb sandwich beams were glued by the $0.2 \mathrm{~mm}$-thick epoxy adhesive film (Shanghai Gongwo, GW-2095) at $120^{\circ} \mathrm{C}$ for $2 \mathrm{~h}$ under nominal pressure of 0.15 MPa, and the CFRP/honeycomb sandwich panel was cured at the same pressure as the aluminum sandwich beams and the same temperature scheme as the unidirectional CFRP laminates. Note that the L-direction sandwich beam denotes the beam whose $x$ direction is parallel to the L-direction of the honeycomb core (Fig. 1a), and the Wdirection sandwich beam is defined similarly.

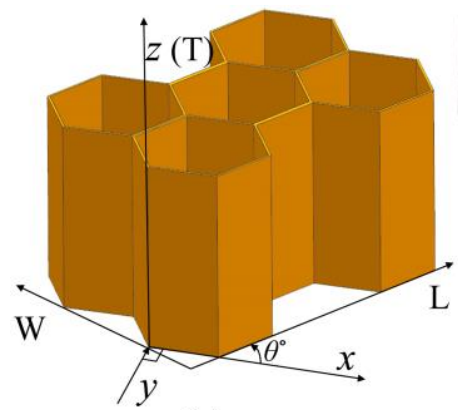

(a)

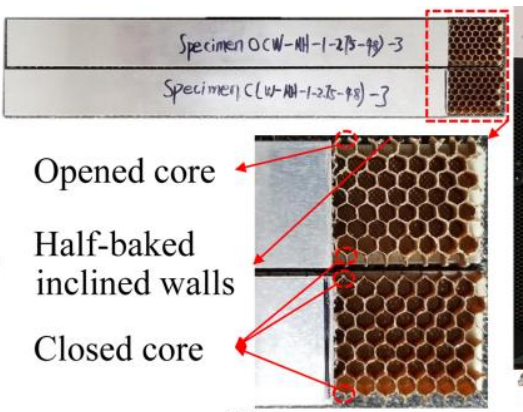

(b)

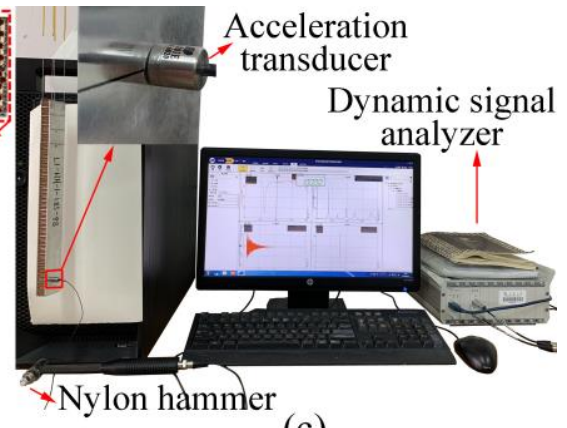

(c)

Fig. 1. Material coordinate, specimen, and corresponding vibration testing equipment: (a) honeycomb coordinate; (b) sandwich specimen and corresponding interior honeycomb cell; (c) modal testing equipment.

\subsection{Specimen dimension and vibration testing}

In order to obtain the damping properties from $500 \mathrm{~Hz}$ to $5000 \mathrm{~Hz}$, specimens with three different lengths were fabricated for each corresponding type of sandwich beams. Since all sandwich beams have the same aluminum skins ( $0.945 \mathrm{~mm}$ in thickness), only dimensions of the honeycomb cores are listed in Table 2. 
To further explore the effect of cutting position, two kinds of typical W-direction honeycomb (NH-1-2.75-48) sandwich beams are also tested in this work. The specimens are shown in Fig. 1b where the cutting position of the interior honeycomb core in the two sandwich specimens is different. Specifically, one has closed honeycomb core on both sides (labeled as Specimens C), and the other has opened honeycomb core on at least one side (labeled as Specimens O). The same dimension configuration as shown in Table 2 is used for these two types of specimens.

There are at least five parallel specimens for each kind of aforementioned beams, and all specimens were placed at least one day at room temperature before testing.

Table 2 Dimensions of honeycomb cores used in sandwich beams.

\begin{tabular}{lcccc}
\hline Core material & Orientation & Length $(\mathrm{mm})$ & Width $(\mathrm{mm})$ & Thickness $(\mathrm{mm})$ \\
\hline \multirow{2}{*}{ NH-1-1.83-48 } & $\mathrm{L}$ & $350 / 310 / 270$ & 32.5 & 12.15 \\
& $\mathrm{~W}$ & $350 / 310 / 270$ & 32.5 & 12.90 \\
NH-1-2.75-48 & $\mathrm{L}$ & $350 / 310 / 270$ & 32.5 & 12.18 \\
& $\mathrm{~W}$ & $350 / 310 / 270$ & 32.5 & 12.18 \\
\hline
\end{tabular}

Fig. 1c. More specifically, the nylon hammer (type LC02) introduces the excitation signals and the acceleration transducer (type 1A801E) with a weight of $1 \mathrm{~g}$ collects the response signals. Then, the captured signals are digitalized and processed by a dynamic signal analyzer (type DH5299N). Finally, the frequency response functions (the ratio of the response signal to the excitation signal after fast Fourier transform) are obtained.

All specimens were suspended by the thin wires in order to reduce the effect of clamping boundaries as much as possible [13]. In addition, the excitation position and the 
response position were unified for all specimens. The maximum frequency resolution adopted in this work was $0.391 \mathrm{~Hz}$, which was determined indirectly by setting the value of sampling frequency $(12.8 \mathrm{kHz})$ and the number of sampling points (32768). Among the settable frequency resolutions, this frequency resolution was sufficient to ensure that all interested peaks of frequency response functions were recorded well. Ten times effective excitations were carried on each excitation point based on the coherence functions, and the average frequency response curves were used to calculate the modal loss factors and natural frequencies. Specifically, the natural frequencies are the frequencies corresponding to the resonance peaks, and the modal loss factors are calculated based on the half-power bandwidth method.

\section{Mathematical model and principle of experimental/numerical method}

\subsection{Mathematical model}

In this work, the damping values of the honeycomb core are derived from the modal loss factors of the sandwich structure. According to the modal strain energy method, the modal loss factor can be defined as modal dissipated energy per unit modal strain energy. For the honeycomb sandwich structure, the thickness ratio of the honeycomb core to the aluminum skin is relatively large, and the in-plane moduli of the honeycomb core are relatively small $[6,14]$. Therefore, the modal strain energy of the honeycomb sandwich structure is mainly contributed by the in-plane strain energy of the skin and the transverse shear strain energy of the core. The $r$ th modal loss factor of the sandwich structure can be calculated by Eq. (1) [14].

$$
\eta_{\mathrm{SW}}^{r}\left(\theta^{\circ}\right)=\frac{\Delta U_{\mathrm{skin}}^{r}+\Delta U_{\mathrm{hc}}^{r}}{U_{\mathrm{t}}}=\frac{\eta_{\mathrm{skin}} U_{\mathrm{skin}}^{r}}{U_{\mathrm{t}}}+\frac{\eta_{\mathrm{WT}} U_{\mathrm{WT}}^{r}}{U_{\mathrm{t}}}+\frac{\eta_{\mathrm{LT}} U_{\mathrm{LT}}^{r}}{U_{\mathrm{t}}}
$$


where $\theta$ is the angle between the $x$-direction and L-direction of honeycomb core (Fig. 1a); $\Delta U_{\text {skin }}$ and $\Delta U_{\mathrm{hc}}$ are the dissipated energy of the skins and the core, respectively; $\eta_{\mathrm{sw}}$ and $U_{\mathrm{t}}$ are the loss factor and strain energy of the integral structure, respectively; $\eta_{\text {skin }}$ and $U_{\text {skin }}$ are the loss factor and strain energy of the skins, respectively; $\eta_{\mathrm{LT}}$ and $\eta_{\mathrm{wT}}$ are the damping coefficients of honeycomb core in the planes $(\mathrm{L}, \mathrm{T})$ and $(\mathrm{W}, \mathrm{T})$, respectively; $U_{\mathrm{LT}}$ and $U_{\mathrm{WT}}$ are the strain energy of honeycomb core in the planes $(\mathrm{L}, \mathrm{T})$ and $(\mathrm{W}, \mathrm{T})$, respectively.

For the aluminum/honeycomb sandwich structure, the modal loss factors of sandwich structure $(\eta \mathrm{sw})$ and aluminum skin $\left(\eta_{\text {skin }}\right)$ in Eq. (1) are obtained by modal testing directly; the modal strain energy of sandwich structure $\left(U_{t}\right)$ and aluminum skin $\left(U_{\text {skin }}\right)$ in Eq. (1) are extracted directly after the modal calculation. Nevertheless, the modal strain energy of the honeycomb core $\left(U_{\mathrm{LT}}\right.$ and $\left.U_{\mathrm{WT}}\right)$ in the material coordinate system cannot be extracted directly; it is calculated by an APDL program in ANSYS 14.5. Specifically, the modal strain energy ( $U_{\mathrm{LT}}$ and $U_{\mathrm{WT}}$ ) was calculated by Eqs. (2) and (3):

$$
\begin{aligned}
& U_{\mathrm{LT}}=\sum_{\text {elements }} U_{\mathrm{LT}}^{e}=\sum_{\text {elements }} \frac{1}{2} \iiint_{e} \frac{\sigma_{\mathrm{LT}}^{2}}{G_{\mathrm{LT}}} \mathrm{d} x \mathrm{~d} y \mathrm{~d} z \\
& U_{\mathrm{WT}}=\sum_{\text {elements }} U_{\mathrm{WT}}^{e}=\sum_{\text {elements }} \frac{1}{2} \iiint_{e} \frac{\sigma_{\mathrm{WT}}^{2}}{G_{\mathrm{WT}}} \mathrm{d} x \mathrm{~d} y \mathrm{~d} z
\end{aligned}
$$

where the $\sigma_{\mathrm{LT}}$ and $\sigma_{\mathrm{WT}}$ are the node shear stresses in planes $(\mathrm{L}, \mathrm{T})$ and $(\mathrm{W}, \mathrm{T})$, respectively. On the basis of the first shear deformation theory (FSDT), these transverse shear stresses have a quadratic relationship with the element thickness (z) [15]. The integral form of these shear stresses in the plane $(x, y)$ depends on the specific type of element. In this work, SHELL 181 which was used to model the honeycomb sandwich by Arunkumar et al. [16] is adopted in this work, and the accuracy of this element is 
governed by the FSDT. The expression of transverse shear stresses of SHELL 181 with the element thickness can be calculated as Eq. (4):

$$
\sigma_{p k}=a_{p k}(x, y) z^{2}+b_{p k}(x, y), \quad p=\mathrm{LT}, \mathrm{WT}
$$

where $k$ is the number of the element. Substituting the node shear stress values $\left(\sigma_{\mathrm{xz}}\right.$ or $\left.\sigma_{\mathrm{yz}}\right)$ on the upper and lower surfaces of this element into Eq. (4), the corresponding coefficients ( $a_{\mathrm{k}}$ and $b_{\mathrm{k}}$ ) can be obtained. For Shell 181, the stress or strain in the element is independent of the in-plane coordinates. Then, the transverse shear strain energy $\left(U_{\mathrm{LT}}\right.$ and $U_{\mathrm{WT}}$ ) of honeycomb core can be obtained by substituting the Eq. (4) to Eqs. (2) and (3), respectively. The TSM ( $G_{\mathrm{LT}}$ and $\left.G_{\mathrm{WT}}\right)$ of the honeycomb core in Eqs. (2) and (3) are obtained by the experimental/numerical method in the next section.

\subsection{Principle of experimental/numerical method}

Since the specimen size and boundary conditions have been determined, the natural frequencies of the aluminum/honeycomb sandwich structure mainly depend on the inplane mechanical properties of the aluminum skins and TSM ( $G_{\mathrm{LT}}$ and $\left.G_{\mathrm{WT}}\right)$ of the honeycomb core. The mechanical parameters of 1060 commercial-purity aluminum are given in Table 1, and it was confirmed that these parameters were scarcely affected by the frequency [10].

For the L-direction sandwich beam, the relatively thick straight walls in L-direction are the primary force-bearing structure. These relatively thick walls are parallel to the shear stress direction, which means that the out-of-plane bending modal natural frequencies of the L-direction beam highly depend on $G_{\mathrm{LT}}$. Moreover, sensitivity analysis shows that the natural frequencies of the L-direction sandwich beam are scarcely affected by the $G_{\mathrm{WT}}$. Therefore, among the material parameters that determine the natural 
frequencies of L-direction honeycomb sandwich beam, the unique unknown parameter $G_{\mathrm{LT}}$ can be obtained by fitting the calculated and experimental natural frequencies. For the L-direction sandwich beam with a fixed length, the $G_{\mathrm{LT}}$ of honeycomb core is sequentially changed to make the difference of the first five-order natural frequencies between the calculation and experiment be less than $0.5 \%$ [17]. Then, the values of $G_{\mathrm{LT}}$ at corresponding natural frequencies are obtained. Subsequently, the transverse shear strain energy, $U_{\mathrm{LT}}$, is calculated by means of ANSYS 14.5 , and the damping values of $\eta_{\mathrm{LT}}$ are further obtained by Eq. (1). Similarly, the frequency-dependent $G_{\mathrm{WT}}$ and $\eta_{\mathrm{WT}}$ can be obtained from the modal parameters of the $\mathrm{W}$-direction sandwich beam.

\section{Results and discussion}

\subsection{Evaluation of specimen size for the measurement of TSM and damping values}

To evaluate the needed specimen size, the effect of cutting position on the properties of the W-direction aluminum/NH-1-2.75-48 sandwich beams is explored in this section. Fig. 2 reports the $G_{\mathrm{WT}}$ and $\eta_{\mathrm{WT}}$ of NH-1-2.75-48. For TSM, $G_{\mathrm{WT}}$ of Specimens C is about $14 \%$ higher than that of the Specimens O, which is mainly caused by the different cutting positions. As shown in Fig. 1b and Fig. 2a, there are eight pairs of intact inclined walls in the width direction in Specimens C while seven in Specimens O. Furthermore, there are some of the half-baked inclined walls on the side edge of Specimens O. On the one hand, one side of these incomplete inclined walls is free. On the other hand, they suffer certain damage inevitably when cut, which means that their ability to carry stress is limited [18]. For the transverse shear deformation of the W-direction sandwich beam, the shear stress in honeycomb core is almost entirely borne by the inclined walls [19]. The number of 
unbroken inclined walls of the former is $14.29 \%$ more than that of the latter, which shows good consistency with the difference of $G_{\mathrm{WT}}$.
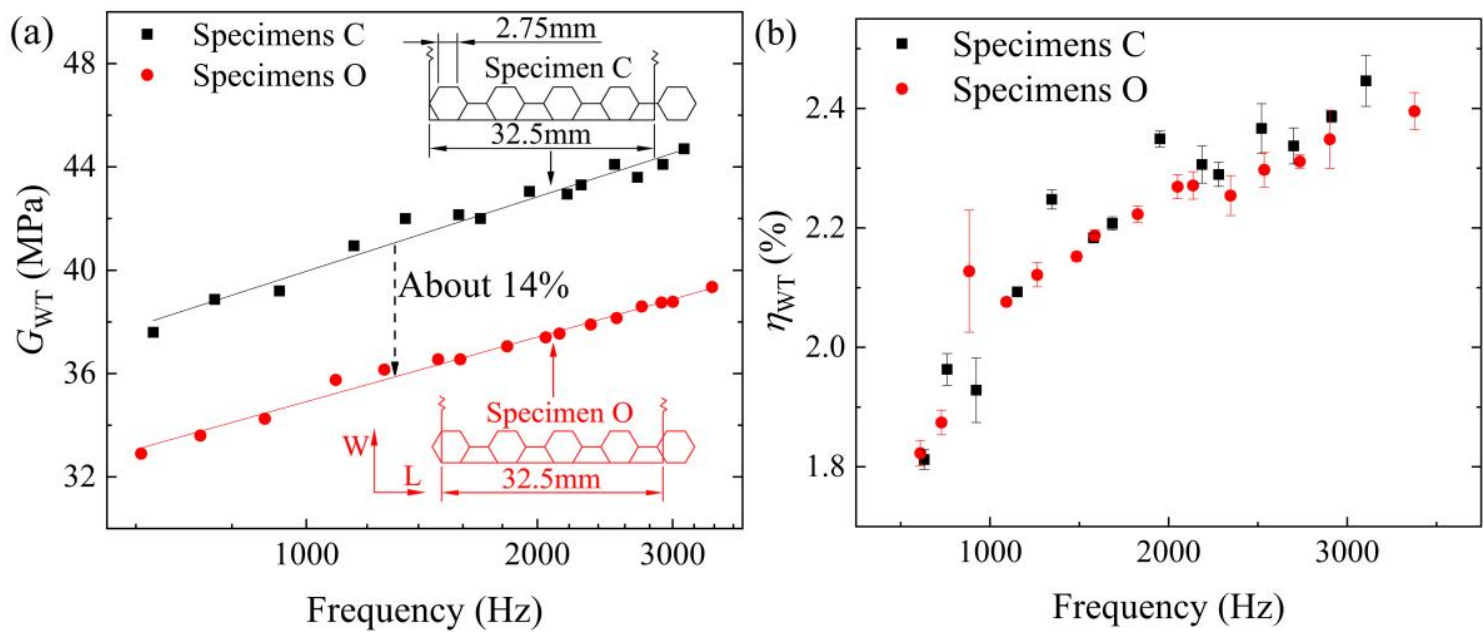

Fig. 2. TSM and damping values of NH-1-2.75-48 with different cutting positions: (a) $G_{\mathrm{WT}}$; (b) $\eta \mathrm{wT}$.

According to the above experimental results and theoretical analysis in the previous section, the difference of $G_{\mathrm{WT}}$ depends on the difference in the number of intact inclined walls. Similarly, the difference in the number of relatively thick straight walls determines the difference of $G_{\mathrm{LT}}$. Therefore, for the L- and W-directions honeycomb cores (Fig. 3), the maximum differences introduced by the cutting position among the test TSM of parallel specimens are $1 /\left(m_{1}-1\right)$ and $1 /\left(m_{2}-1\right)$, respectively, where $m_{1}$ and $m_{2}$ are the maximum number of straight walls and pairs of intact inclined walls that can be contained in the $\mathrm{W}$-direction and L-direction, respectively. The corresponding specimen widths $w_{1}$ and $w_{2}$ are shown in Fig. 3, where $l$ is the cell side length of the honeycomb core. Therefore, the maximum differences of $G_{\mathrm{LT}}\left(\mathrm{D} G_{\mathrm{LT}}\right)$ and $G_{\mathrm{WT}}\left(\mathrm{D} G_{\mathrm{WT}}\right)$ among the test specimens can be evaluated as $\mathrm{DG}_{\mathrm{LT}} \approx \operatorname{sqrt}(3) l /\left(2 w_{1}\right)$ and $\mathrm{D} G_{\mathrm{WT}} \approx 3 l /\left(2 w_{2}-l\right)$, respectively, which reflects the discrete degree of the test results. 


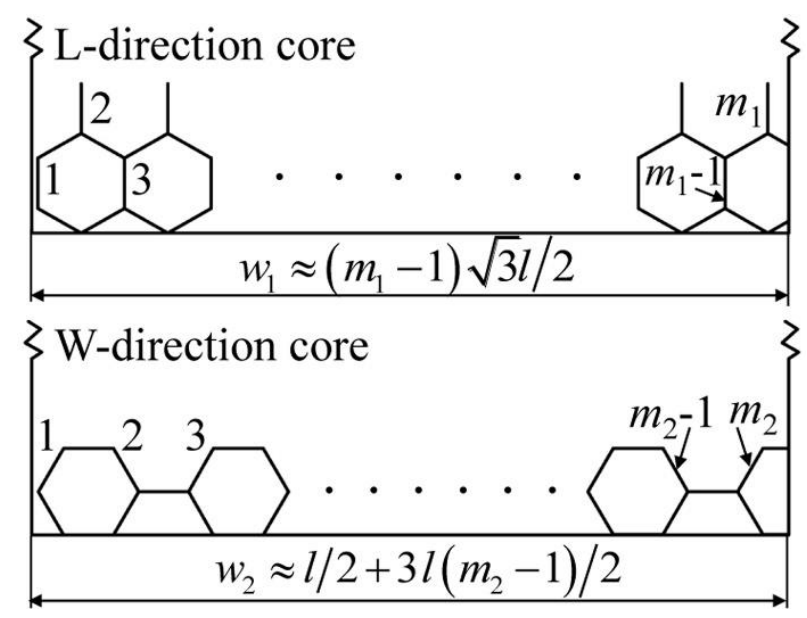

Fig. 3. L-direction and W-direction honeycomb cores.

It needs to be pointed out that the above analysis mainly focuses on the case where the shear deformation of the honeycomb core in the plane $(\mathrm{L}, \mathrm{T})$ or plane $(\mathrm{W}, \mathrm{T})$ is dominant, which is not limited by the specific testing method. In other words, for the measurement of TSM of honeycomb cores with different cell side lengths, the stipulation about the geometry of test specimens in standard ASTM C273/C273M [20] can be further refined. For example, for a honeycomb core with a relatively large cell side length (e.g., $4.5 \mathrm{~mm}$ ), if $\mathrm{D} G_{\mathrm{LT}}$ and $\mathrm{D} G_{\mathrm{WT}}$ are to be controlled within $10 \%$, the specimen widths used to test the $G_{\mathrm{LT}}$ and $G_{\mathrm{WT}}$ should be not less than $39.84 \mathrm{~mm}$ and $68 \mathrm{~mm}$, respectively.

No matter how many intact inclined walls are deformed in the bending mode of the sandwich structure, the dissipated energy per unit strain energy is unchanged. Therefore, it is easy to understand the interesting phenomenon (Fig. 2b) that the damping values of the honeycomb cores are roughly equivalent. It indicates that the process of the obtaining of damping values has a relatively low requirement for specimen size. In order to keep a proper length-width ratio about 10:1 [21] and width at the same time, the length of the specimen may be more than $500 \mathrm{~mm}$, and the corresponding equipment for specimen fabrication is unavailable for us. Therefore, a compromise that the specimen with the 
width of $32.5 \mathrm{~mm}$ continues to be used, and simple arithmetic averages of $G_{\mathrm{WT}}$ for NH-12.75-48 between the two aforementioned situations are used in the following sections. All these values will be validated in Section 4.2.3.

\subsection{Effect of cell side length on the fundamental orthotropic damping coefficients}

\subsubsection{TSM of honeycomb cores}

After determining the size of the specimen, the TSM of honeycomb cores are obtained by fast fitting the calculated and experimental modal natural frequencies. The frequency-dependent $G_{\mathrm{LT}}$ and $G_{\mathrm{WT}}$ of honeycomb cores are reported in Fig. 4, where the $G_{\mathrm{WT}}$ of NH-1-1.83-48 is roughly equal to that of NH-1-2.75-48, and the $G_{\mathrm{LT}}$ of the former is about $10 \%$ higher than the latter. A similar trend was shown in reference [8] in which the $G_{\text {LT }}$ of A1-64-3 is about 7.6\% higher than that of A1-64-5 by shear testing, where the three symbols denote Nomex honeycomb, bulk density, and cell size, respectively.
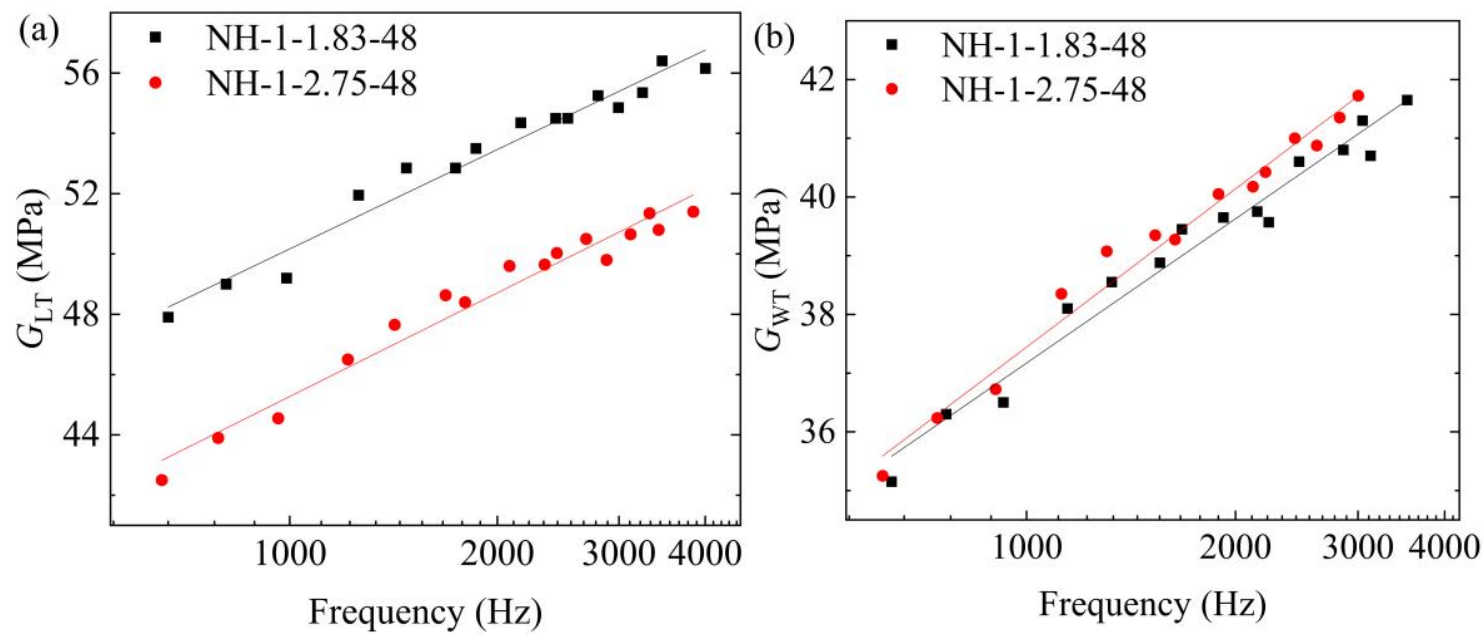

Fig. 4. TSM of different honeycomb cores: (a) $G_{\mathrm{LT}}$; (b) $G_{\mathrm{WT}}$.

\subsubsection{Effect of cell side length on the orthotropic damping coefficients}


The damping values of honeycomb cores with different cell side lengths are calculated by Eq. (1) after obtaining the TSM of honeycomb cores. These damping values under different frequencies are reported in Fig. 5, where the $\eta_{\text {LT }}$ of NH-1-1.83-48 is slightly higher than that of NH-1-2.75-48 (Fig. 5a) while the $\eta$ wT of the former are about $7 \%$ higher than that of the latter (Fig. 5b). The damping values of Nomex honeycomb cores with different cell side lengths and volume densities tested by Adams and Bacon [8] under the quasi-static conditions are $1.61 \%-1.78 \%$, which are relatively lower than the damping values between $600 \mathrm{~Hz}$ and $4000 \mathrm{~Hz}$ in this work.
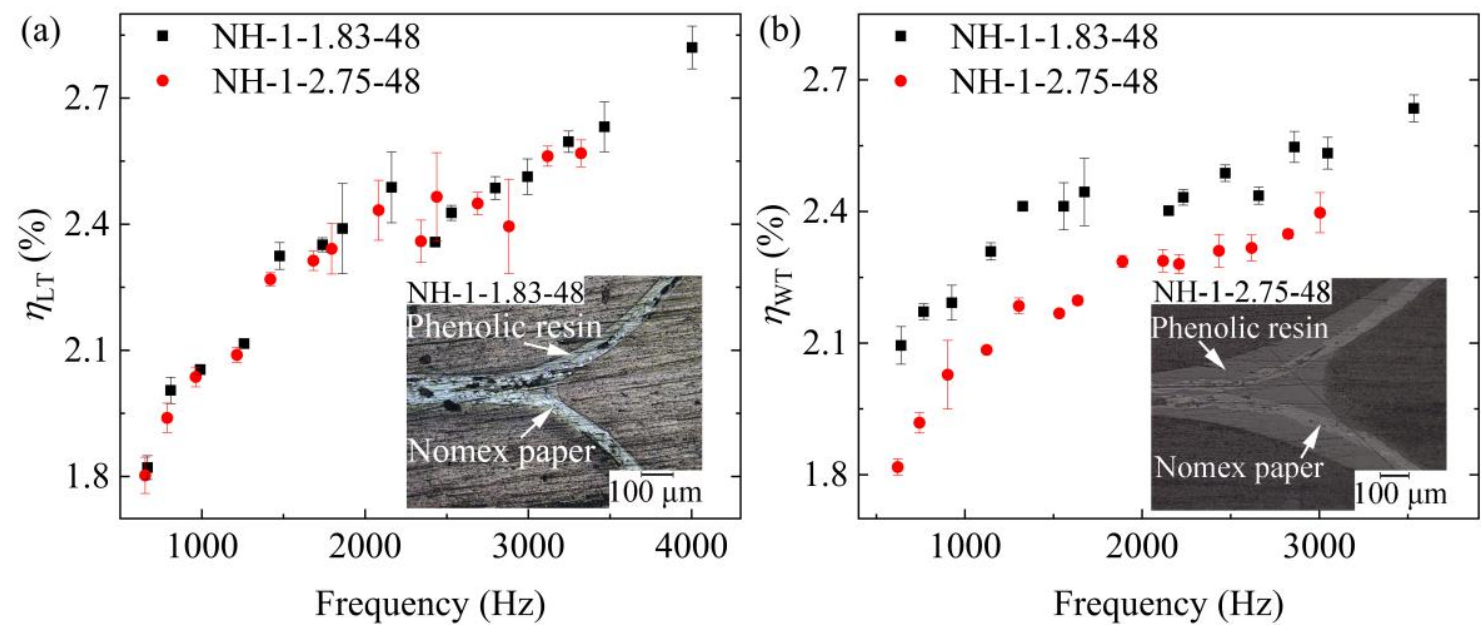

Fig. 5. Damping values of different honeycomb cores: (a) $\eta_{\mathrm{LT}}$; (b) $\eta_{\mathrm{wT}}$.

For the honeycomb core in the plane $(\mathrm{L}, \mathrm{W})$, the length of Nomex paper per unit area is about $8 \times \operatorname{sqrt}(3) /(9 l)$. Therefore, the length of the interface (including the interface between the Nomex paper and phenolic resin as well as the bonding interface between Nomex paper and Nomex paper) per unit area in $\mathrm{NH}-1-1.83-48$ is about 50\% longer than that of NH-1-2.75-48. This means that when the cell side length of honeycomb core changes from $2.75 \mathrm{~mm}$ to $1.83 \mathrm{~mm}$, the mass of Nomex paper increases by about $50 \%$. In order to maintain the same volume density $\left(48 \mathrm{~kg} / \mathrm{m}^{3}\right)$, the phenolic resin reduces the same mass. 
The dissipated energy of honeycomb core during deformation is mainly contributed by the microfiber friction of Nomex paper, the intermolecular friction of phenolic resin, and the interfacial friction. For the transverse shear deformation of honeycomb core in the plane (L, T), most of the total strain energy is stored in the straight walls [19]. As shown in Fig. 5a, the damping difference of $\eta_{\mathrm{LT}}$ between the two types of honeycomb cores is very small although the mass fraction of each component varies greatly. Since the straight walls are parallel to the plane (L, T) and the Coulomb friction in the interfacial phase is relatively hard to take place, relatively small difference of $\eta_{\mathrm{LT}}$ implies that the damping value of Nomex paper is approximately equal to that of the phenolic resin. In comparison with the L-direction core, the interfacial friction is relatively easy to take place in the transverse shear deformation of inclined walls in the $\mathrm{W}$-direction core. Therefore, the damping value of $\eta_{\mathrm{wT}}$ increases by about $7 \%$ as shown in Fig. $5 \mathbf{b}$ when the length of interface increases by 50\%, which means that the damping value of the interfacial phase is higher than that of the other two components. The relatively large difference of $\eta_{\mathrm{LT}}$ also reveals that the difference in energy consumption of the interfacial phase in different honeycomb orientations of leads to the orthotropic damping properties of honeycomb core.

Compared with NH-12.75-48, NH-1-1.83-48 has relatively higher $G_{\mathrm{LT}}$ and $\eta_{\mathrm{WT}}$ in the entire interested frequency range. Overall, the $G_{\mathrm{WT}}$ and $\eta_{\mathrm{wT}}$ of the two kinds of honeycomb cores are roughly equivalent.

\subsubsection{Validation of the TSM and damping values}

In order to validate the obtained mechanical and damping values above, adopting these parameters, modal parameters of $45^{\circ}$-direction aluminum/honeycomb sandwich 
beams and CFRP/honeycomb sandwich panel (300 $\mathrm{mm}$ in length and $200 \mathrm{~mm}$ in width) are calculated and compared with the corresponding experimental results. In the vibration modes of the aforementioned sandwich structures, both of the $U_{\mathrm{WT}}^{r} / U_{\mathrm{t}}$ and $U_{\mathrm{LT}}^{r} / U_{\mathrm{t}}$ cannot be neglected, which means that the parameters $\left(G_{\mathrm{LT}}, G_{\mathrm{WT}}, \eta_{\mathrm{LT}}\right.$, and $\left.\eta_{\mathrm{WT}}\right)$ of the honeycomb core can play a role in the vibration modes of the corresponding honeycomb sandwich structures. Thus, the parameters of the honeycomb core can be validated by comparing the calculated and experimental modal parameters of these sandwich structures. Taking NH-1-2.75-48 as an example, the results are reported in Fig. $\mathbf{6}$.
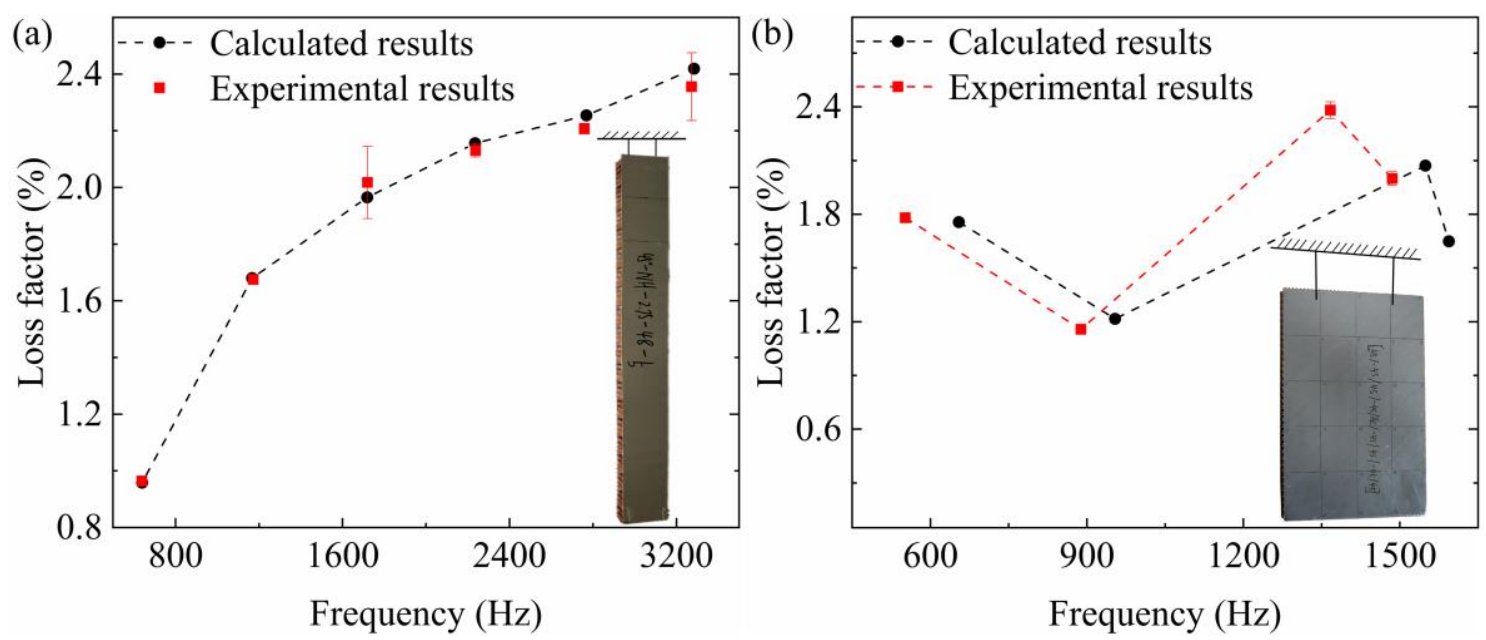

Fig. 6. Parameters validation by: (a) $45^{\circ}$-direction aluminum/honeycomb sandwich beam; (b) CFRP/honeycomb sandwich panel.

As shown in Fig. 6a, the first six-order calculated modal natural frequencies and loss factors match with the experimental results well for the $45^{\circ}$-direction sandwich beam, and the corresponding maximum deviations are $-0.47 \%$ (the second mode) and $2.69 \%$ (the sixth mode), respectively. It indicates that the obtained mechanical and damping values of the honeycomb core are reliable. 
In the case of CFRP/honeycomb sandwich panel, the thin skins suffer a certain degree of mechanical properties loss during the one-step forming process, due to its relatively low forming pressure and insufficiently smooth surface. Therefore, the calculated natural frequencies are always larger than the corresponding experimental results as shown in Fig. 6b. As for the modal loss factor, the damping values of the main components are obtained from the beam with a relatively small surface. Considering that the structure with a relatively large surface has relatively high additional air damping [24], when these parameters were used to calculate the modal loss factors of the sandwich panel, the measured modal loss factors should be greater than the calculated results in theory. However, the larger the skin stiffness is, the larger the strain energy ratio (SER) of the core is. Hence, the overestimated mechanical properties of CFRP skin result in the overestimated SER of the core. Since the damping property of the core is higher than the skin at relatively low frequencies, the overestimated SER of the core further leads to the overvalued damping values of the sandwich structure at relatively low-order modes. Therefore, the deviations between the calculated and experimental loss factors at relatively low-order modes are relatively small.

\subsection{Effect of beam orientation on the orthotropic damping behavior}

According to the aforementioned results, the orthotropic damping properties of honeycomb core mainly result from the difference of damping contribution of the interfacial phase in different directions. Therefore, the angle-ply aluminum/honeycomb sandwich beams with a length of $350 \mathrm{~mm}$ are investigated to reveal the effect of beam orientation on the specific orthotropic damping behaviors. Using the validated frequencydependent fundamental damping coefficients and TSM, the first three bending and 
torsional modal loss factors with different beam orientations are obtained by Eq. (1). The results are reported in Fig. 7, where the $0^{\circ}$-direction beam is the L-direction sandwich beam and the $90^{\circ}$-direction beam is the $\mathrm{W}$-direction sandwich beam. Since the results of these two types of sandwich structures are similar to each other, only the results of aluminum/NH-1-2.75-48 are shown for simplicity.
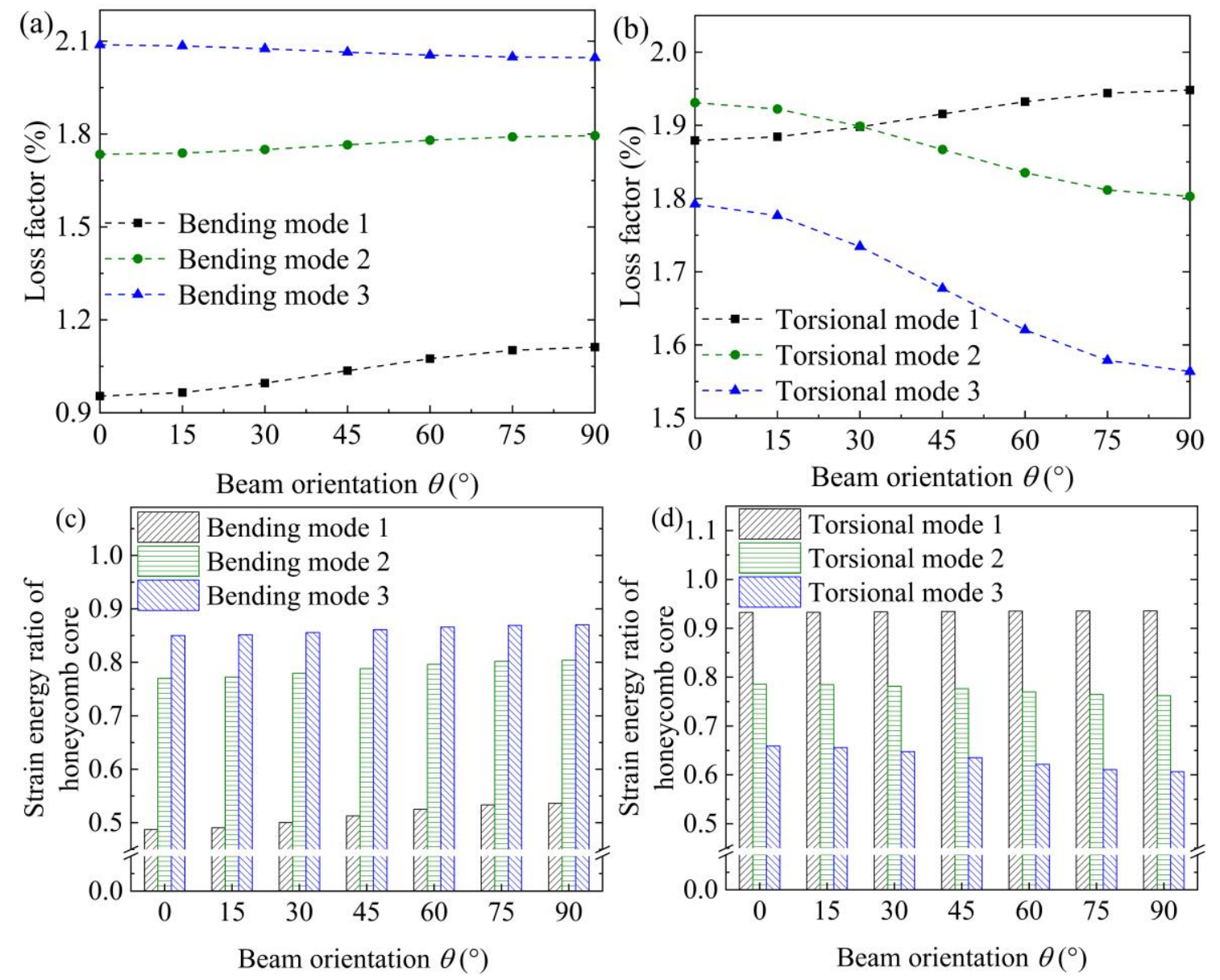

Fig. 7. Variation of modal loss factors of honeycomb sandwich beams and SER of honeycomb core with beam orientation $\theta$ : (a) first three bending modal loss factors; (b) first three torsional modal loss factors; (c) SER in the first three bending modes; (d) SER in the first three torsional modes. 
The damping values of the honeycomb sandwich structure depend on both the damping coefficient and the SER of each component. As shown in Fig. 7c and 7d, the SER of honeycomb core in the relatively low-order modes (including the first bending, second bending, and first torsional modes) increases gradually when the beam orientation changes from $0^{\circ}$ to $90^{\circ}$. As reported in Fig. 5, the W-direction $\left(90^{\circ}\right)$ honeycomb core has relatively higher damping values than that of the L-direction $\left(0^{\circ}\right)$ honeycomb core at a relatively low frequency. Consequently, these relatively low-order modal loss factors increase with the increasing $\theta$. The situation is opposite at a relatively high frequency where the $\eta_{\mathrm{LT}}$ is about $6 \%$ higher than $\eta_{\mathrm{WT}}$ (Fig. 5), which leads to the result that the remaining relatively high-order modal loss factors decline with the increasing $\theta$.

The damping value of honeycomb core increases with the frequency as shown in Fig. 5, and relatively high vibration mode has a relatively high natural frequency. Therefore, the damping coefficient of the honeycomb core is high at a relatively high vibration mode. For the out-of-plane bending modes, the SER of honeycomb core increases with the increasing vibration mode (Fig. 7c). Thus, the bending modal damping value increases with the increasing vibration mode rapidly, as shown in Fig. 7a. For the torsional modes, the SER of the honeycomb core has a nearly linear decline with the increasing vibration mode (frequency). In the meanwhile, the damping value of the honeycomb core increases approximately logarithmically with the frequency (Fig. 5). Therefore, the torsional modal damping values of the honeycomb sandwich beam increase first and then decrease. But the high-order modal loss factors decrease with the increasing beam orientation as mention above, which leads to the inconsistent damping behavior for the sandwich beam with different beam orientations as shown in Fig. 7b. 
The honeycomb materials are usually not used alone in practical engineering applications, so the performance of the honeycomb sandwich structure needs to be considered. For the investigated honeycomb sandwich beams, the thickness ratio of core to the skin is larger than ten, and the elastic modulus of the skin is about three orders of magnitude higher than that of the investigated honeycomb cores. Therefore, as shown in Eq. (5), the main excellent mechanical properties like the static bending stiffness $(D)$ are mainly dependent on the mechanical properties of the skins and the physical dimension of each component [23].

$$
D=\sum_{q} 2 E_{q} w \int_{t_{q-1}}^{t_{q}} z^{2} \mathrm{~d} z=\frac{E_{\mathrm{s}} w h_{\mathrm{s}}^{3}}{6}+\frac{E_{\mathrm{s}} w h_{\mathrm{s}}\left(h_{\mathrm{c}}+h_{\mathrm{s}}\right)^{2}}{2}+\frac{E_{\mathrm{c}} w h_{\mathrm{c}}^{3}}{12}
$$

where $E_{\mathrm{s}}$ and $E_{\mathrm{c}}$ are the elastic moduli of skin and honeycomb core, respectively; $h_{\mathrm{s}}$ and $h_{\mathrm{c}}$ are the thickness of skin and honeycomb core, respectively; $w$ is the width of the honeycomb sandwich structure. Since the skin materials and physical dimensions are the same for all the sandwich beams with different beam orientations, the static bending stiffness of these sandwich beams is approximately equal to each other. Therefore, when the static bending stiffness and damping property of the sandwich beam should be considered at the same time, the $90^{\circ}$-direction sandwich beam might be the candidate at a relatively low frequency and the $0^{\circ}$-direction sandwich beam might be the candidate at a relatively high frequency $(1200-4000 \mathrm{~Hz})$.

\section{Summary}

The frequency-dependent orthotropic damping properties of honeycomb composites are investigated by means of the experimental/numerical method. The effects of cutting position, cell side length and beam orientation are discussed. Several conclusions are summarized as follows: 
(1) For the measurement of TSM of honeycomb core, the relationship between the theoretical maximum difference and specimen width is quantified. For the measurement of damping values, the requirement for the specimen size is relatively low, which shows relatively high robustness.

(2) Without increasing any weight, within a certain range of cell side length, the smaller the cell side length of the honeycomb core is, the higher the $G_{\mathrm{LT}}$ and $\eta_{\mathrm{wT}}$ the honeycomb core has. Truly, in the meantime, the cost will increase with the increasing mass fraction of Nomex paper.

(3) The anisotropic damping properties of the honeycomb core are mainly caused by the different energy dissipation contributions of the interfacial phase to honeycomb core in different directions. For the application scenarios where the bending stiffness and damping property of the sandwich beam need to be considered first, the W-direction and L-direction sandwich beams will be the candidate at a relatively low frequency and a relatively high frequency, respectively.

\section{Acknowledgements}

The authors are thankful to Doctor Xufeng Zhang (AVIC Composites Co., Ltd.) for supplying the Nomex honeycomb materials.

\section{Funding}

This work was supported by the National Natural Science Foundation of China [Grant No. 51973105] and the Special Research Foundation of China Civil Aircraft [Grant No. MJ-2015-H-G-103]. 


\section{References}

[1] S. Xie, H. Wang, C. Yang, H. Zhou, Z. Feng, Mechanical properties of combined structures of stacked multilayer Nomex ${ }^{\circledR}$ honeycombs, Thin-Walled Struct. 151 (2020) 106729.

[2] T.N. Bitzer, Honeycomb Technology: Materials, Design, Manufacturing, Applications and Testing, Chapman \& Hall, New York, 1997.

[3] Q. Zhang, X. Yang, P. Li, G. Huang, S. Feng, C. Shen, B. Han, X. Zhang, F. Jin, F. $\mathrm{Xu}, \mathrm{T} . J . \mathrm{Lu}$, Bioinspired engineering of honeycomb structure - Using nature to inspire human innovation, Progress in Materials Science 74 (2015) 332-400.

[4] M. Matter, T. Gmür, J. Cugnoni, A. Schorderet, Numerical-experimental identification of the elastic and damping properties in composite plates, Compos. Struct. 90(2) (2009) 180-187.

[5] M. Matter, T. Gmür, J. Cugnoni, A. Schorderet, Identification of the elastic and damping properties in sandwich structures with a low core-to-skin stiffness ratio, Compos. Struct. 93(2) (2011) 331-341.

[6] B.X. Rébillat M, Measurement of relevant elastic and damping material properties in sandwich thick plates, J. Sound. Vib. 330(25) (2011) 6098-6121.

[7] D. Jiang, D. Zhang, Q. Fei, S. Wu, An approach on identification of equivalent properties of honeycomb core using experimental modal data, Finite Elem. Anal. Des. 90 (2014) 84-92.

[8] R.D. Adams, M.R. Maheri, The dynamic shear properties of structural honeycomb materials, Compos. Sci. Technol. 47(1) (1993) 15-23.

[9] G. Knapp, G. Oreski, G. Pinter, Method to characterize the damping behavior of thin 
passively constrained layer laminates using dynamic mechanical analysis (DMA) in shear mode, Polym. Testing. 42 (2015) 215-224.

[10] Y. Zhou, Q. Wang, Y. Guo, Y. Xu, X. Yi, Y. Jia, Effect of phenolic resin thickness on frequency-dependent dynamic mechanical properties of Nomex honeycomb cores, Composites Part B: Engineering 154 (2018) 285-291.

[11] D.A. Pereira, T.A.M. Guimarães, H.B. Resende, D.A. Rade, Numerical and experimental analyses of modal frequency and damping in tow-steered CFRP laminates, Compos. Struct. 244 (2020) 112190.

[12] Z. Wang, R. Tian, Aluminum alloy and its processing manual, Central South University Press, Changsha, 1989.

[13] M. Rueppel, J. Rion, C. Dransfeld, C. Fischer, K. Masania, Damping of carbon fibre and flax fibre angle-ply composite laminates, Compos. Sci. Technol. 146 (2017) 1-9.

[14] M.R. Maheri, R.D. Adams, J. Hugon, Vibration damping in sandwich panels, Journal of Materials Science 43 (2008) 6604-6618.

[15] A.M. Berthelot Jean-Marie, Sefrani Youssef, A.E. Mahi, Damping analysis of composite materials and structures, Composite Structures 85(3) (2008) 189-204.

[16] M.P. Arunkumar, M. Jagadeesh, J. Pitchaimani, K.V. Gangadharan, M.C.L. Babu, Sound radiation and transmission loss characteristics of a honeycomb sandwich panel with composite facings: Effect of inherent material damping, Journal of Sound and Vibration 383 (2016) 221-232.

[17] M. Assarar, A.E. Mahi, J.M. Berthelot, Evaluation of the dynamic properties of PVC foams under flexural vibrations, Compos. Struct. 94(6) (2012) 1919-1931.

[18] A. Shahdin, L. Mezeix, C. Bouvet, J. Morlier, Y. Gourinat, Fabrication and 
mechanical testing of glass fiber entangled sandwich beams: A comparison with honeycomb and foam sandwich beams, Composite Structures 90(4) (2009) 404-412.

[19] R.S. Wang, J. Wang, Modeling of honeycombs with laminated composite cell walls, Compos. Struct. 184 (2018) 191-197.

[20] ASTM C273/C273M-16, Standard test method for shear properties of sandwich core materials, 2016.

[21] R.D. Adams, M.R. Maheri, Dynamic flexural properties of anisotropic fibrous composite beams, Compos. Sci. Technol. 50(4) (1994) 497-514.

[22] M. Wesolowski, E. Barkanov, Air damping influence on dynamic parameters of laminated composite plates, Measurement 85 (2016) 239-248.

[23] H.Y. Kim, W. Hwang, Effect of debonding on natural frequencies and frequency response functions of honeycomb sandwich beams, Compos. Struct. 55(1) (2002) 5162. 


\section{Figure captions:}

Fig. 1. Material coordinate, specimen, and corresponding vibration testing equipment: (a) honeycomb coordinate; (b) sandwich specimen and corresponding interior honeycomb cell; (c) modal testing equipment.

Fig. 2. TSM and damping values of NH-1-2.75-48 with different cutting positions: (a) $G_{\mathrm{WT}}$; (b) $\eta \mathrm{wT}$.

Fig. 3. L-direction and W-direction honeycomb cores.

Fig. 4. TSM of different honeycomb cores: (a) $G_{\mathrm{LT}}$; (b) $G_{\mathrm{WT}}$.

Fig. 5. Damping values of different honeycomb cores: (a) $\eta_{\mathrm{LT}}$; (b) $\eta_{\mathrm{wT}}$.

Fig. 6. Parameters validation by: (a) $45^{\circ}$-direction aluminum/honeycomb sandwich beam; (b) CFRP/honeycomb sandwich panel.

Fig. 7. Variation of modal loss factors of honeycomb sandwich beams and SER of honeycomb core with beam orientation $\theta$ : (a) first three bending modal loss factors; (b) first three torsional modal loss factors; (c) SER in the first three bending modes; (d) SER in the first three torsional modes. 